\title{
Los briófitos de la Estancia Cerro Paine, Parque Nacional Torres del Paine, Magallanes, Chile
}

\author{
JUAN LARRAÍN ${ }^{1}$ y NELSON BAHAMONDE²
}

\begin{abstract}
Summary: The bryophytes of Estancia Cerro Paine, Torres del Paine National Park, Magallanes, Chile. We present a checklist of non-vascular plants for Estancia Cerro Paine, a private land located in the heart of Torres del Paine National Park. The list includes two hornworts, 29 liverworts and 93 moss species, making a total of 124 bryophyte species. We report 32 new bryophyte records for Última Esperanza Province, 11 for Magallanes Region, and three new records for Chile (Bryum apiculatum, Tortella fragilis and Valdonia microcarpa). The known distribution limits of six taxa are extended to the north, whereas for seven taxa the distribution limits are extended to the south. This is the first bryophyte species list made within the limits of Torres del Paine National Park.
\end{abstract}

Key words: Bryophyta, Marchantiophyta, Anthocerotophyta, sub-Antarctic forests, Biosphere Reserve.

Resumen: Se presenta una lista de plantas no vasculares para la Estancia Cerro Paine, predio particular ubicado en el centro del Parque Nacional Torres del Paine. La lista incluye dos antocerotes, 29 hepáticas y 93 especies de musgos, totalizando 124 especies de briófitos. Se reportan 32 nuevos registros para la provincia de Última Esperanza, 11 para la Región de Magallanes y tres nuevos registros para Chile (Bryum apiculatum, Tortella fragilis y Valdonia microcarpa). Se extienden hacia el norte los límites de distribución de seis taxones, mientras que los de siete se extienden hacia el sur. Este es el primer listado de briófitos realizado dentro de los límites del Parque Nacional Torres del Paine.

Palabras clave: Bryophyta, Marchantiophyta, Anthocerotophyta, bosques subantárticos, Reserva de la Biósfera.

\section{INTRODUCCIÓN}

Los briófitos (musgos, hepáticas y antocerotes) juegan un rol importante en los ecosistemas templados al ser una fracción importante de la biomasa fotosintética, fijando importantes cantidades de carbono atmosférico y liberando oxígeno (Schofield, 1985), y por absorber y retener los minerales disueltos en las aguas de lluvia permitiendo la incorporación de éstos en el ecosistema y disminuyendo su lavado hacia los ríos y mares (Rieley et al., 1979). Son importantes

1 Instituto de Biología, Facultad de Ciencias, Pontificia Universidad Católica de Valparaíso, Campus Curauma, Av. Universidad 330, Curauma, Valparaíso, Chile. musgoschiloe@gmail.com.

${ }^{2}$ AMA Torres del Paine, Estancia Cerro Paine s.n., comuna Torres del Paine, Chile. reguladores del ciclo del agua en bosques y turberas (Rieley et al., 1979; van Breemen, 1995; van Seters \& Price, 2001) y protegen los suelos de la erosión causada por viento, agua y temperaturas extremas (Ross et al., 1984). Los briófitos proveen hogar y protección a un sinnúmero de pequeños invertebrados como insectos, arácnidos, rotíferos, nemátodos, tardígrados, moluscos y anélidos (Gerson, 1982; Suren, 1993), y son utilizados por aves y pequeños mamíferos como material de nidificación (Calvelo et al., 2006; Torres-Dowdall et al., 2007; Honorato et al., 2013).

La conspicua abundancia, elevada diversidad y alto grado de endemismo de los briófitos de los bosques del sur de Sudamérica ha llamado la atención de los especialistas desde hace más de dos siglos. Si bien aún falta mucho que avanzar en el conocimiento de la flora briofítica de Chile, casi toda la atención ha sido enfocada en los briófitos 
del extremo sur, generando una buena base de conocimiento de la diversidad de la Región de Magallanes. Casi todos los primeros exploradores de la flora briofítica de Chile realizaron colecciones en los alrededores del Estrecho de Magallanes o en Tierra del Fuego, y ya antes del comienzo del siglo XIX se contaba con varias colecciones de briófitos provenientes de Magallanes (Gunckel, 1971; Larraín \& Marticorena, 2009). Éstas luego dieron paso a importantes contribuciones sobre la flora briofítica de la región (Hooker \& Wilson, 1844; Wilson \& Hooker, 1847; Müller, 1885; Cardot, 1901, 1908; Dusén, 1903, 1905a, 1905b, 1906a, 1906b; Cardot \& Brotherus, 1923; Roivainen, 1937; Matteri, 1985; Buck \& Goffinet, 2010). Todo este conocimiento se ha traducido en la existencia de varias guías de campo para aficionados que destacan la flora briofítica y liquénica del sur de la región (e.g. Goffinet et al., 2012; Rozzi et al., 2012).

Sin embargo, y a pesar de ser la Región de Magallanes la que mayor número de especies de briófitos posee a lo largo de Chile (Müller, 2009a; Hässel de Menéndez \& Rubies, 2009), vastas áreas de la región aún permanecen escasamente exploradas, en especial las áreas de difícil acceso o sitios donde los briófitos no son tan abundantes o llamativos. El Parque Nacional Torres del Paine (PNTP) es un ejemplo de esta situación, ya que las zonas fácilmente accesibles son relativamente secas y las áreas más remotas del parque son solamente accesibles por senderos de caminata, lo que limita las posibilidades de realizar una prospección en detalle. Las colecciones briológicas realizadas en el parque son escasas y recientes, destacando la expedición japonesa de 1981 y la expedición alemana "Bryo Austral 2" de 2001 con colecciones realizadas por Hironori Deguchi, y Wolfgang Frey y Friederike Schaumann respectivamente. Ambas expediciones, junto con otras menores, recolectaron briófitos en diferentes áreas del PNTP. Algunos duplicados de dichas colecciones se encuentran disponibles en el herbario del Museo Nacional de Historia Natural (SGO), aunque la mayoría de éstas se encuentran en herbarios extranjeros. A pesar de esto aún no existe un listado de especies de briófitos para el PNTP.

En este contexto surgió la iniciativa de hacer una prospección de la flora de briófitos de la Estancia Cerro Paine (ECP), como un punto de partida para el futuro estudio de la indocumentada diversidad briofítica del parque nacional más visitado de Chile. El PNTP se ha transformado en un área vulnerable a los impactos de la actividad humana debido a los reiterados incendios forestales y la creciente actividad turística (Vidal, 2012). Las consecuencias del último incendio ocurrido en Torres del Paine el verano del año 2011-2012 impulsaron la incorporación del concepto de "restauración ecológica" para dirigir los trabajos que apuntan a recuperar lo dañado en el parque. Sin embargo, los esfuerzos de implementar trabajos de restauración se complican cuando se carece de una base de información acerca de la totalidad de los componentes de los sistemas biológicos anteriores a los disturbios. Determinar el éxito de la restauración es igualmente complicado sin contar con la información previa necesaria. El conocimiento existente acerca de la brioflora del área es dispersa y de difícil acceso para no especialistas.

\section{Material y Método}

\section{Área de estudio}

El PNTP es un área perteneciente al Sistema Nacional de Áreas Protegidas del Estado de Chile, creada en 1959 y declarada reserva de la biósfera por la UNESCO el año 1978 (CONAF, 2007). En su interior se ubica el predio particular Estancia Cerro Paine (lat. 5058’26”S, long. 7252'50”W), el único terreno privado dentro de los límites del parque y que no se encuentra bajo la administración de la Corporación Nacional Forestal (CONAF, 2007). La estancia tiene una superficie de 4400 hectáreas, representando solo el $2 \%$ de la superficie total del PNTP (CONAF, 2007). Se ubica en la Región de Magallanes y Antártica Chilena, Provincia de Última Esperanza, comuna Torres del Paine (Fig. 1).

Esta propiedad fue adquirida en el año 1976 con fines ganaderos, actividad económica actualmente reemplazada en su totalidad por el turismo de naturaleza remota. Este predio contiene segmentos considerables de los senderos turísticos que utilizan los visitantes que van al parque para recorrer el perímetro del macizo de Torres del Paine y es paso obligado para realizar el recorrido del sendero que conduce al mirador de la base de las Torres 


\section{J. Larraín y N. Bahamonde - Briofitos de Torres del Paine, Chile}

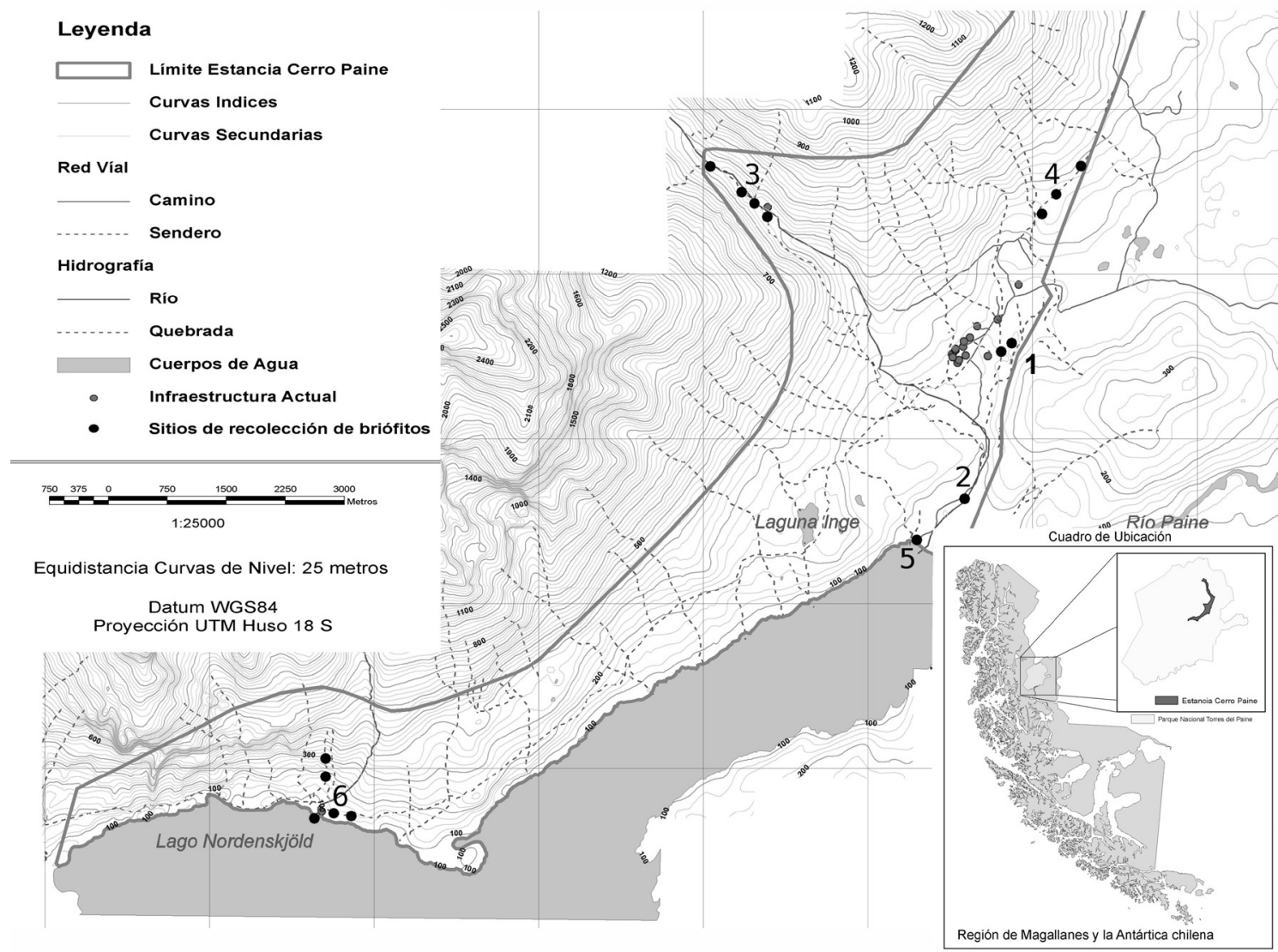

Fig. 1. Ubicación geográfica de Estancia Cerro Paine (ECP) en la región de Magallanes y en el Parque Nacional Torres del Paine (PNTP). Los números corresponden a las localidades de muestreo explicadas en el texto.

del Paine. Si bien esta porción de territorio está separada del terreno correspondiente al PNTP, constituye un continuo de los componentes naturales del área. Al igual que el resto del parque, ya ha sido caracterizada a través de líneas de base que han realizado inventarios de las especies de plantas y animales que habitan en el lugar (CONAF, 2007). En cuanto a la caracterización florística, los listados resultantes de las investigaciones dentro del parque y los inventarios de la ECP se enfocan en la descripción de las plantas vasculares, desconociéndose la riqueza de especies de las plantas no vasculares o briófitas (Arroyo et al., 1992; Boelcke et al., 1985; Correa, 1971-1999; Dollenz, 1991a, 1991b; Dollenz \& Ivanovic, 1996; Domínguez, 2003; Domínguez et al., 2004, 2006; Pisano, 1974; Vidal, 2007).
La ECP posee áreas características y representativas del PNTP como son:

-Humedales: áreas con mal drenaje que albergan avifauna y especies vegetales tales como cariofiláceas y juncáceas, además de bosquetes enanos de Nothofagus antarctica (G.Forst.) Oerst. (ñire).

-Matorral xerófito: se encuentra en las zonas más áridas, vegetación baja y abierta, con especies representativas como Mulinum spinosum Pers. (mata barrosa), Mulguraea tridens (Lag.) N.O'Leary \& P.Peralta (mata negra) y Senecio patagonicus Hook. \& Arn.

-Matorral mesófito: en la periferia de los bosques o en zonas más húmedas, con especies representativas tales como, Berberis microphylla G.Forst. (calafate), Discaria chacaye (G.Don) Tortosa (espino blanco), 
Empetrum rubrum Vahl ex Willd. (murtilla) y especies arbóreas de tamaño pequeño tales como Nothofagus anctartica, N. betuloides (Mirb.) Oerst. (coigüe de Magallanes) y N. pumilio (Poepp. et Endl.) Krasser (lenga).

-Bosque deciduo: dominados por las especies Nothofagus pumilio y $N$. antarctica, los que pueden alcanzar alturas considerables y generan un sotobosque abierto, fácil de penetrar.

-Bosque perenne: ubicados en quebradas y en zonas húmedas, dominados por Nothofagus betuloides y Drimys winteri J.R.Forst. \& Forst. (canelo), con presencia de Maytenus magellanica (Lam.) Hook.f. (leñadura) y un sotobosque denso y rico en briófitos.

El clima del área estudiada se caracteriza por tener temperaturas relativamente bajas, sin variaciones extremas entre invierno y verano, siendo menos de $15^{\circ} \mathrm{C}$ la temperatura máxima del mes más cálido (enero), y la temperatura mínima media para el mes más frío (julio) nunca inferior a los $-2,5^{\circ} \mathrm{C}$. Uno de los aspectos que definen el clima en el área en estudio es la ausencia de una estación seca. Existe un claro gradiente pluviométrico tanto longitudinal como altitudinal, las que se concentran en los meses de verano y comienzos del otoño.

\section{Trabajo de Campo}

Entre los días 16-19 de enero de 2015 y 14-18 de enero de 2016 se realizaron recorridos de muestreo aleatorio de la brioflora al interior de la ECP, considerando que los resultados de la exploración de esta área constituirían el primer listado de plantas no vasculares para la comuna de Torres del Paine donde se encuentra el PNTP. Este trabajo preliminar eventualmente permitiría justificar la investigación de este grupo de plantas en la totalidad del área del PNTP, con el fin de generar las instancias que permitan describir en forma integral la riqueza de especies vegetales contenidas en el área protegida incluyendo a ECP. Esto resulta relevante considerando la connotación de conservación que representa este territorio y el desconocimiento de la riqueza específica de la totalidad de las especies de plantas, lo que deja fuera una cantidad aún desconocida de musgos, hepáticas, antocerotes, líquenes y hongos que habitan en el área y que probablemente requieran de una consideración especial para su conservación.

Las localidades visitadas fueron las siguientes (ver Fig. 1):
1) Sector Las Torres, en sendero interpretativo frente a Hotel Las Torres (números 38465-38485, 39364-39380, 39448-39456).

2) Sector Las Torres, por camino entre hotel Las Torres y Lago Nordenskjöld (números 3848638491).

3) Por sendero a la base de las torres, pasando por Campamento Chileno (números 38492-38562 + 27399).

4) Por el sendero entre sector Las Torres y Serón, en bosque de Nothofagus pumilio (números 3856338606, 39381-39418).

5) Afloramientos rocosos en el embarcadero en el extremo E del Lago Nordenskjöld (números 38607-38615).

6) Alrededores del Campamento Los Cuernos (números 38616-38644, 39419-39447).

El siguiente listado, representa el primer inventario de plantas no vasculares para el área, el cual incrementa la riqueza de especies vegetales reportadas a la fecha, en un número considerable de especies que hasta ahora no han sido considerados para la comuna, el PNTP y la ECP.

\section{Resultados}

Se realizaron un total de 295 colecciones de briófitos en los nueve días de trabajo de campo. Fueron encontrados un total de 124 especies de briófitos correspondientes a dos especies de antocerotes, 29 hepáticas y 93 especies de musgos, pertenecientes a 38 familias y 76 géneros. Se encontraron 32 nuevos registros para la provincia de Última Esperanza, 11 para la Región de Magallanes $\mathrm{y}$ tres nuevos registros para Chile (Bryum apiculatum Schwägr., Tortella fragilis (Hook. \& Wilson) Limpr. y Valdonia microcarpa (Mitt.) Ochyra). Seis taxones extienden sus límites de distribución hacia el norte, mientras que siete lo extienden hacia el sur. La numeración corresponde al herbario personal del primer autor. Esta información se resume en la Tabla 1. Todos los especímenes están depositados en el herbario de la Universidad de Concepción (CONC) con duplicados de las hepáticas en el Field Museum de Chicago (F).

Resulta importante de destacar aquí el hallazgo de tres nuevos registros para la flora de musgos de Chile. Bryum apiculatum supuestamente presenta una distribución pantropical extendiéndose hacia 
Tabla 1. Lista de especies de briófitos encontrados en la Estancia Cerro Paine. Un asterisco $\left(^{*}\right)$ indica

los nuevos registros para la Provincia Última Esperanza, dos asteriscos $\left({ }^{* *}\right)$ indica nuevos registros

para la Región de Magallanes, y tres $\left({ }^{* * *}\right)$ indican nuevo registro para Chile. EL= extensión de límite de distribución, (S) por el sur, (N) por el norte.

\begin{tabular}{|c|c|c|c|}
\hline Taxón & Familia & Número de colección & EL \\
\hline \multicolumn{4}{|l|}{ ANTOCEROTES } \\
\hline Nothoceros fuegiensis (Steph.) J.C.Villarreal & Dendrocerotaceae & $38516,38556,39439 A$ & \\
\hline *Phaeomegaceros skottsbergii (Steph.) R.J.Duff \& al. & Dendrocerotaceae & 38641 & \\
\hline \multicolumn{4}{|l|}{ HEPÁTICAS } \\
\hline $\begin{array}{l}\text { Acrobolbus flavicans (J.J.Engel \& Grolle) } \\
\text { Brisco \& J.J.Engel }\end{array}$ & Acrobolbaceae & 39397 & \\
\hline${ }^{* *}$ Aneura pinguis (L.) Dumort. & Aneuraceae & $39374 \mathrm{~A}$ & \\
\hline${ }^{*}$ Cephaloziella varians (Gottsche) Steph. & Cephaloziellaceae & 39428B & $\mathrm{N}$ \\
\hline Chiloscyphus durus (Steph.) Hässel & Lophocoleaceae & 38595 & \\
\hline $\begin{array}{l}{ }^{*} \text { Clasmatocolea rigens (Hook.f. \& Taylor) } \\
\text { J.J.Engel \& R.M.Schust. }\end{array}$ & Lophocoleaceae & 38522B, 38541, 38574, 39396 & \\
\hline${ }^{*}$ Clasmatocolea vermicularis (Lehm.) Grolle & Lophocoleaceae & $\begin{array}{l}\text { 38532B, 38558, 38596B, 38617, } \\
38631,39427,39442\end{array}$ & \\
\hline $\begin{array}{l}\text { Cryptochila grandiflora } \\
\text { (Lindenb. \& Gottsche) Grolle }\end{array}$ & Jungermanniaceae & 39429B & \\
\hline Frullania fertilis De Not. & Frullaniaceae & 38535 & \\
\hline Isotachis spegazziniana C.Massal. & Balantiopsaceae & 39438 & \\
\hline *Leptoscyphus cf. magellanicus (Gola) Hässel & Lophocoleaceae & 38530,38628 & $\mathrm{~N}$ \\
\hline $\begin{array}{l}\text { Leptoscyphus chiloscyphoideus } \\
\text { (Lindenb. ex Lehm.) Gottsche }\end{array}$ & Lophocoleaceae & $38529,38563,39429 A, 39432$ & \\
\hline Lethocolea radicosa (Lehm. \& Lindenb.) Grolle & Acrobolbaceae & 38583 & \\
\hline${ }^{* *}$ Lophocolea dentiflora Steph. & Lophocoleaceae & 39407 & $\mathrm{~S}$ \\
\hline $\begin{array}{l}\text { Lophocolea textilis (Hook.f. \& Taylor) } \\
\text { Taylor ex Gottsche, Lindenb. \& Nees }\end{array}$ & Lophocoleaceae & 38544 & \\
\hline *Lophozia excisa (Dicks.) Dumort. & Lophoziaceae & 38588 & \\
\hline *Marchantia polymorpha L. & Marchantiaceae & 39448 & \\
\hline Metzgeria decipiens (C.Massal.) Schiffn. & Metzgeriaceae & 38587 & \\
\hline Metzgeria epiphylla A.Evans & Metzgeriaceae & $38538,38575,39403,39412$ & \\
\hline *Noteroclada confluens Taylor ex Hook.f. \& Wilson & Pelliaceae & 38616 & \\
\hline Pachyglossa austrigena (Hook.f. \& Taylor) L.Söderstr. & Lophocoleaceae & 38525 & \\
\hline Pachyglossa boveana (C.Massal.) L.Söderstr. & Lophocoleaceae & 38643 & \\
\hline Plagiochila flava Steph. & Plagiochilaceae & 38534 & \\
\hline Plagiochila mitis Inoue & Plagiochilaceae & $38582,38583 b$ & \\
\hline $\begin{array}{l}\text { Pseudolepicolea quadrilaciniata } \\
\text { (Sull.) Fulford \& J.Taylor }\end{array}$ & Pseudolepicoleaceae & 39443 & \\
\hline Reboulia hemisphaerica (L.) Raddi & Aytoniaceae & 38540 & \\
\hline Riccardia diversiflora A.Evans & Aneuraceae & $38557,38622,38625,39374 B$ & \\
\hline Riccardia granulata (Steph.) A.Evans & Aneuraceae & 38591 & \\
\hline *Temnoma patagonicum R.M.Schust. & Pseudolepicoleaceae & 38623 & \\
\hline *Temnoma pilosum (A.Evans) R.M.Schust. & Pseudolepicoleaceae & $38584,39436,39437$ & \\
\hline \multicolumn{4}{|l|}{ MUSGOS } \\
\hline Acrocladium auriculatum (Mont.) Mitt. & Lembophyllaceae & 38504 & \\
\hline **Amblystegium varium (Hedw.) Lindb. & Amblystegiaceae & 38618 & $\mathrm{~S}$ \\
\hline
\end{tabular}




\begin{tabular}{|c|c|c|c|}
\hline Taxón & Familia & Número de colección & EL \\
\hline *Andreaea regularis Müll. Hal. & Andreaeaceae & 38561,38639 & \\
\hline *Aulacomnium palustre (Hedw.) Schwägr. & Aulacomniaceae & 38479,39373 & \\
\hline Bartramia ithyphylloides Schimp. ex Müll.Hal. & Bartramiaceae & $\begin{array}{l}38473,38548,38571,38589 \\
39379\end{array}$ & \\
\hline Bartramia mossmaniana Müll.Hal. & Bartramiaceae & 38507 & \\
\hline Bartramia patens Brid. & Bartramiaceae & 38498,38510 & \\
\hline Bartramia stricta Brid. & Bartramiaceae & 38472,39378 & \\
\hline Blindia magellanica Schimp. & Seligeriaceae & $\begin{array}{l}\begin{array}{l}38555,38568,38576,38594, \\
38596 A, 38621 A, 38630,39431, \\
39440\end{array}\end{array}$ & \\
\hline $\begin{array}{l}\text { Brachytheciastrum paradoxum (Hook.f. \& Wilson) } \\
\text { Ignatov \& Huttunen }\end{array}$ & Brachytheciaceae & $38509,38546,39394$ & \\
\hline $\begin{array}{l}\text { **Brachythecium collinum (Schleich. ex Müll.Hal.) } \\
\text { Schimp. }\end{array}$ & Brachytheciaceae & 38604,39393 & S \\
\hline Brachythecium praelongum Schimp. ex Müll.Hal. & Brachytheciaceae & 38489,38497 & \\
\hline Brachythecium subplicatum (Hampe) A.Jaeger & Brachytheciaceae & 38478,39449 & \\
\hline Breutelia integrifolia (Taylor) A.Jaeger & Bartramiaceae & $\begin{array}{l}38480,38482,38552,38559 \\
38599,38619 A, 38626\end{array}$ & \\
\hline Bryoerythrophyllum recurvirostrum (Hedw.) P.C.Chen & Pottiaceae & $38524,38609,38621 \mathrm{~B}$ & \\
\hline Bryum algovicum Sendtn. ex Müll.Hal. & Bryaceae & 38560 & \\
\hline${ }^{* * * B}$ Bryum apiculatum Schwägr. & Bryaceae & 38614,39369 & \\
\hline Bryum laevigatum Hook.f. \& Wilson & Bryaceae & $38492,38554,38620,39435$ & \\
\hline Bryum orbiculatifolium Cardot \& Broth. & Bryaceae & 38613 & \\
\hline Bryum perlimbatum Cardot & Bryaceae & $38500,38567,39417,39434$ & \\
\hline $\begin{array}{l}\text { Bryum pseudotriquetrum (Hedw.) } \\
\text { G.Gaertn., B.Mey. \& Scherb. }\end{array}$ & Bryaceae & 38608 & \\
\hline Ceratodon purpureus (Hedw.) Brid. & Ditrichaceae & $\begin{array}{l}38470,38483,38491,38615 \\
38629,39370,39377\end{array}$ & \\
\hline Catagonium nitens (Brid.) Cardot & Catagoniaceae & 39411 & \\
\hline Chorisodontium aciphyllum (Hook.f. \& Wilson) Broth. & Dicranaceae & 38502 & \\
\hline Cratoneuropsis chilensis (Lorentz) Ochyra & Amblystegiaceae & $\begin{array}{l}38484,38486,38496,38550 \\
38592,39372\end{array}$ & \\
\hline $\begin{array}{l}\text { Dendroligotrichum squamosum } \\
\text { (Hook.f. \& Wilson) Cardot }\end{array}$ & Polytrichaceae & 39446 & \\
\hline Dicranella campylophylla (Taylor) A.Jaeger & Dicranellaceae & 39395,39426 & \\
\hline Dicranella hookeri (Müll.Hal.) Cardot & Dicranellaceae & $\begin{array}{l}38481,38493,38607,38611 \mathrm{~A}, \\
39452\end{array}$ & \\
\hline Didymodon andreaeoides Cardot \& Broth. & Pottiaceae & 38603,39415 & \\
\hline $\begin{array}{l}\text { Didymodon australasiae (Hooker \& Greville) } \\
\text { R.H.Zander }\end{array}$ & Pottiaceae & 39413 & \\
\hline Distichium capillaceum (Hedw.) Bruch \& Schimp. & Ditrichaceae & $38518,38570 \mathrm{~B}$ & \\
\hline Ditrichum hyalinum (Mitt.) Kuntze & Ditrichaceae & 38553 & \\
\hline $\begin{array}{l}\text { Drepanocladus longifolius (Wilson ex Mitt.) } \\
\text { Broth. ex Paris }\end{array}$ & Amblystegiaceae & 39371 & \\
\hline Drepanocladus polygamus (Schimp.) Hedenäs & Amblystegiaceae & 39450,39451 & \\
\hline $\begin{array}{l}{ }^{* *} \text { Dryptodon austrofunalis (Müll.Hal.) } \\
\text { Ochyra \& Żarnowiec }\end{array}$ & Grimmiaceae & 39410 & $\mathrm{~S}$ \\
\hline Encalypta ciliata Hedw. & Encalyptaceae & 38600,39367 & \\
\hline
\end{tabular}




\section{J. Larraín y N. Bahamonde - Briofitos de Torres del Paine, Chile}

\begin{tabular}{|c|c|c|c|}
\hline Taxón & Familia & Número de colección & EL \\
\hline Eurhynchium fuegianum Cardot & Brachytheciaceae & 38585,39385 & \\
\hline Goniobryum subbasilare (Hook.) Lindb. & Rhizogoniaceae & 38543 & \\
\hline Grimmia humilis Mitt. & Grimmiaceae & 38467 & \\
\hline${ }^{*}$ Grimmia longirostris Hook. & Grimmiaceae & 39425 & \\
\hline${ }^{* *}$ Grimmia navicularis Herzog & Grimmiaceae & 38569,38636 & $\mathrm{~S}$ \\
\hline Grimmia pulvinata (Hedw.) Sm. & Grimmiaceae & $\begin{array}{l}38610,38612,39368,39376 \\
39433\end{array}$ & \\
\hline Grimmia trichophylla Grev. & Grimmiaceae & $39390,39399 A$ & \\
\hline Hennediella antarctica (Ångström) Ochyra \& Matteri & Pottiaceae & 38528,38533 & \\
\hline Holodontium strictum (Hook.f. \& Wilson) Ochyra & Rhabdoweisiaceae & 39447 & \\
\hline $\begin{array}{l}\text { Hymenodontopsis mnioides (Hook.) } \\
\text { N.E.Bell, A.E.Newton \& D.Quandt }\end{array}$ & Aulacomniaceae & 38531 & \\
\hline Hymenoloma crispulum (Hedw.) Ochyra & Rhabdoweisiaceae & $38517,38536,39422$ & \\
\hline Isopterygiopsis pulchella (Hedw.) Z.Iwats. & Plagiotheciaceae & 38564 & \\
\hline Leptobryum pyriforme (Hedw.) Wilson & Meesiaceae & 39453 & \\
\hline Lepyrodon lagurus (Hook.) Mitt. & Lepyrodontaceae & 38508 & \\
\hline *Orthotrichum anomalum Hedw. & Orthotrichaceae & 39384 & $\mathrm{~N}$ \\
\hline Orthotrichum anaglyptodon Cardot \& Broth. & Orthotrichaceae & $38476,38577,39391$ & \\
\hline **Orthotrichum araucarieti Müll.Hal. & Orthotrichaceae & $38505,39387,39401 \mathrm{~B}$ & S \\
\hline Orthotrichum assimile Müll.Hal. & Orthotrichaceae & $\begin{array}{l}38466,38547,39364,39365 \\
39456\end{array}$ & \\
\hline Orthotrichum ludificans Lewinsky & Orthotrichaceae & $38542 A, 38573,38578$ & \\
\hline${ }^{* *}$ Orthotrichum pariatum Mitt. & Orthotrichaceae & 39389B & S \\
\hline Orthotrichum rupestre Schleich. ex Schwägr. & Orthotrichaceae & 39388, 39389A, 39399E & \\
\hline *Philonotis polymorpha (Müll.Hal.) Kindb. & Bartramiaceae & 38495 & \\
\hline Philonotis scabrifolia (Hook.f. \& Wilson) Braithw. & Bartramiaceae & $38519 A$ & \\
\hline Philonotis vagans (Hook.f. \& Wilson) Mitt. & Bartramiaceae & 27399 & \\
\hline Plagiomnium ellipticum (Brid.) T.J.Kop. & Mniaceae & 39455 & \\
\hline $\begin{array}{l}\text { Platyneuron praealtum (Mitt.) } \\
\text { Ochyra \& Bednarek-Ochyra }\end{array}$ & Dicranaceae & 38506 & \\
\hline Pohlia cruda (Hedw.) Lindb. & Mniaceae & $\begin{array}{l}38485,38513,38519 B, 38593 \\
38597\end{array}$ & \\
\hline Pohlia nutans (Hedw.) Lindb. & Mniaceae & 38551,39441 & \\
\hline Pohlia wahlenbergii (F.Weber \& D.Mohr) A.L.Andrews & Mniaceae & $38523,38640,39409,39430$ & \\
\hline Polytrichadelphus magellanicus (Hedw.) Mitt. & Polytrichaceae & 38549 & \\
\hline Polytrichum juniperinum Hedw. & Polytrichaceae & 38490,38579 & \\
\hline Polytrichum piliferum Hedw. & Polytrichaceae & 38566 & \\
\hline Pseudocrossidium crinitum (Schultz) R.H.Zander & Pottiaceae & $\begin{array}{l}38468,38511,38512,38515 C \\
38532 A, 38580,38601,39380, \\
39423\end{array}$ & \\
\hline Racomitrium didymum (Mont.) Lorentz & Grimmiaceae & $38471,38572,38627$ & \\
\hline Racomitrium geronticum Müll.Hal. & Grimmiaceae & 39445 & \\
\hline Racomitrium lamprocarpum (Müll.Hal.) A.Jaeger & Grimmiaceae & 38634,38638 & \\
\hline Racomitrium ptychophyllum (Mitt.) Mitt. & Grimmiaceae & 38562 & \\
\hline Racomitrium subcrispipilum (Müll.Hal.) A.Jaeger & Grimmiaceae & 38520,38632 & \\
\hline Sanionia uncinata (Hedw.) Loeske & Amblystegiaceae & 39444 & \\
\hline Schistidium andinum (Mitt.) Herzog & Grimmiaceae & $\begin{array}{l}38515 A, 38539,38635,39375 \\
39400,39402 A, 39405,39406, \\
39414,39419,39424\end{array}$ & \\
\hline
\end{tabular}


Bol. Soc. Argent. Bot. 52 (1) 2017

\begin{tabular}{|c|c|c|c|}
\hline Taxón & Familia & Número de colección & EL \\
\hline *Schistidium cupulare (Müll.Hal.) Ochyra & Grimmiaceae & $\begin{array}{l}38515 B, 38521,38527,38644, \\
39399 C, 39404,39418,39421\end{array}$ & $\mathrm{~N}$ \\
\hline Schistidium falcatum (Hook.f. \& Wilson) B.Bremer & Grimmiaceae & $38526,38545,39408$ & \\
\hline${ }^{*}$ Schistidium praemorsum (Müll.Hal.) Herzog & Grimmiaceae & 38521A, 38606, 38642 & $\mathrm{~N}$ \\
\hline Scorpidium revolvens (Sw.) Rubers & Amblystegiaceae & 38477 & \\
\hline Syntrichia andersonii (Ångström) R.H.Zander & Pottiaceae & $\begin{array}{l}38474,38475,38633,39366, \\
39416\end{array}$ & \\
\hline Syntrichia antarctica (Hampe) R.H.Zander & Pottiaceae & 38605 & \\
\hline *Syntrichia lithophila (Dusén) Ochyra \& R.H.Zander & Pottiaceae & 38488,38602 & $\mathrm{~N}$ \\
\hline Syntrichia princeps (De Not.) Mitt. & Pottiaceae & 38469 & \\
\hline Syntrichia robusta (Hook. \& Grev.) R.H.Zander & Pottiaceae & $38586,38619 B, 38624,38637$ & \\
\hline *Syntrichia saxicola (Cardot) R.H.Zander & Pottiaceae & $\begin{array}{l}\text { 38501, 38503, 38537, } 38565 \text {, } \\
\text { 39392, 39399D, 39420 }\end{array}$ & \\
\hline ***Tortella fragilis (Hook. \& Wilson) Limpr. & Pottiaceae & 39454 & \\
\hline Ulota macrocalycina Mitt. & Orthotrichaceae & $\begin{array}{l}\text { 38514, 38542B, 39386, 39399B, } \\
39401 \mathrm{~A}\end{array}$ & \\
\hline Ulota pygmaeothecia (Müll.Hal.) Kindb. & Orthotrichaceae & 39402B & \\
\hline${ }^{* * \star V a l d o n i a ~ m i c r o c a r p a ~(M i t t .) ~ O c h y r a ~}$ & Seligeraceae & 38570A, 39381, 39382, 39383 & \\
\hline Vittia pachyloma (Mont.) Ochyra & Amblystegiaceae & 38494,38590 & \\
\hline Weissia cf. controversa Hedw. & Pottiaceae & 38487 & \\
\hline “Zygodon magellanicus Dusén ex Malta & Orthotrichaceae & 38522A, 39398 & \\
\hline
\end{tabular}

las zonas templadas del hemisferio norte (Ochi, 1974; tratado allí como Bryum nitens Hook., cf. Ochi, 1980), siendo éste el registro más austral conocido para la especie. Las plantas recolectadas presentan propágulos axilares, inflorescencias masculinas y femeninas, y esporofítos, por lo que la identificación pudo realizarse satisfactoriamente. Este taxón se caracteriza por las hojas lanceoladas y no decurrentes, apiculadas, con la costa levemente excurrente, bordes planos o ligeramente recurvados, y células basales más anchas que las células mediales.

Tortella fragilis era hasta hace algunos años considerada exclusiva del hemisferio norte. Desde entonces la especie ha sido reportada en varias localidades en el hemisferio sur, incluyendo la parte argentina de Tierra del Fuego (Hyvönen, 1991). La poblaciones antárticas de este taxón han sido recientemente descritas y comparadas en detalle con material del hemisferio norte (Ochyra et al., 2008). Nuestra colección de ECP, a pesar de estar estéril, muestra todos los caracteres que distinguen a este taxón de otras especies del género, como los ápices de las hojas deciduos separándose en una parte única, la parte distal de la hoja biestratificada, y la sección transversal de hojas con las bandas ventral y dorsal de estereidas bien desarrollada, de hasta 4-5 capas de células.

Valdonia microcarpa ha sido considerada una especie endémica de la provincia de Kerguelen, incluyendo las islas Príncipe Eduardo, Marion, de la Posesión y Kerguelen (Ochyra, 2003). Es de hecho el único género de musgos endémico de la región subantártica. Está estrechamente emparentado con Blindia Bruch \& Schimp., pero se diferencia por tener una fila de células guía en la sección transversal de la costa, rodeada de bandas de estereidas dorsales y ventrales, células corto-rectangulares rodeando la costa en la parte distal de las hojas, engrosamientos cuticulares longitudinales que le dan un aspecto papiloso a las hojas en sección transversal, y dientes del peristoma densamente granulosos tanto en su cara interna como externa. El material de la ECP sin embargo, se diferencia de las poblaciones de la provincia de Kerguelen en su condición sexual consistentemente dioica, con parches masculinos creciendo separados de los parches femeninos. Es probable que el material encontrado en ECP corresponda a una nueva especie del género Valdonia, lo que podría 


\section{J. Larraín y N. Bahamonde - Briofitos de Torres del Paine, Chile}

ser corroborado con estudios comparativos de la morfología y genética de las poblaciones chilenas con las de islas subantárticas, lo que se escapa del foco de esta contribución.

Adicionalmente, otras 8 especies son aquí reportadas como nuevos hallazgos para la Región de Magallanes. Éstos son: Aneura pinguis (L.) Dumort., especie casi cosmopolita (Schuster, 1992), reportada anteriormente para Chile en las provincias de Valparaíso y Curicó (Hässel \& Rubies, 2009); Amblystegium varium (Hedw.) Lindb. una especie cosmopolita no reportada previamente para Magallanes, cuyos registros previos para Chile son tratados por Müller (2009a) como conespecíficos con Cratoneuropsis relaxa subsp. minor (Wilson \& Hook.f.) Ochyra, opinión no compartida por nosotros; Brachythecium collinum (Schleich. ex Müll.Hal.) Schimp., especie ampliamente distribuida en el hemisferio norte, con dos registros conocidos en Argentina, y recientemente reportado como nuevo para Chile sobre la base de una colección de la Provincia General Carrera (Müller 2009b); Dryptodon austrofunalis (Müll.Hal.) Ochyra \& Żarnowiec (=Grimmia austrofunalis Müll.Hal.), taxón andino que alcanza por el norte las montañas de Venezuela (Muñoz, 1999), y previamente reportada en Chile entre las provincias de Cachapoal y Aisén (Müller, 2009a); Grimmia navicularis Herzog, considerado previamente distribuido en Chile entre las provincias de Petorca y Aisén (Müller, 2009a); Lophocolea dentiflora Steph. (=Chiloscyphus dentiflorus (Steph.) J.J.Engel \& R.M.Schust.), especie endémica del sur de Chile, previamente reportada entre las provincias de Concepción y Llanquihue (Hässel \& Rubies, 2009); Orthotrichum araucarieti Müll. Hal., también recientemente reportado como nuevo para Chile por Müller (2009b) sobre la base de un único ejemplar de los alrededores de Villa Cerro Castillo, en la provincia de General Carrera, en Aisén; y Orthotrichum pariatum Mitt., taxón andino extendiendo su distribución hasta Costa Rica por el norte (Lewinsky 1987), y reportado previamente para Chile solo de la provincia de Talca (Müller, 2009b).

Además de la ya discutida Valdonia microcarpa, tres especies fueron identificadas con algún grado de incertidumbre: Weissia cf. controversa Hedw., Leptoscyphus cf. magellanicus (Gola) Hässel y Aneura pinguis. El primer taxón se encontró estéril por lo que su identidad no puede ser corroborada, sin embargo ésta es la única especie del género conocida para Última Esperanza. Los especímenes identificados como Leptoscyphus cf. magellanicus difieren de las descripciones de este taxón en que los periantos tienen la boca entera, sin dientes. Nuestras colecciones podrían corresponder a una especie no descrita del género Leptoscyphus Mitt., pero aquí lo reportamos como probablemente $L$. magellanicus mientras el material no sea estudiado más en profundidad. El material determinado como Aneura pinguis podría también corresponder a la especie Lobatiriccardia verdoornioides Nebel, Preussing, Schäf.-Verw. \& D.Quandt, recientemente descrita para Ecuador (Preussing et al., 2010). Lamentablemente el espécimen encontrado está estéril, y al momento de observar los oleocuerpos 10 días después de recolectado el material, éstos ya se habían desvanecido, impidiendo la identificación certera de la colección. Dado que el género Lobatiriccardia (Mizut. \& S.Hatt.) Furuki no ha sido aún reportado para Chile, decidimos reportar el material como Aneura Dumort. mientras no tengamos mayor evidencia para pensar que Lobatiriccardia crece en el sur de Sudamérica.

Entre las hepáticas más comunes en la ECP se pueden destacar Clasmatocolea rigens (Hook.f. \& Taylor) J.J.Engel \& R.M.Schust., C. vermicularis (Lehm.) Grolle y Riccardia diversiflora Evans. Estos tres taxones, al igual que la mayoría de las hepáticas encontradas, se limitan a las zonas más húmedas de la estancia, en bordes de arroyos dentro de bosques, o sobre rocas junto a los arroyos. En el caso de los musgos, las especies más comunes en ambientes forestales son Acrocladium auriculatum (Mont.) Mitt., Bartramia mossmaniana Müll. Hal., Brachytheciastrum paradoxum (Hook.f. \& Wilson) Ignatov \& Huttunen, Cratoneuropsis chilensis (Lorentz) Ochyra, Lepyrodon lagurus (Hook.) Mitt., Schistidium cupulare (Müll.Hal.) Ochyra y Syntrichia anderssonii (Ångström) R.H.Zander. En ambientes más xéricos las especies de musgos más comunes son Bartramia ithyphylloides Schimp. ex Müll.Hal., Grimmia pulvinata (Hedw.) Sm., Orthotrichum assimile Müll.Hal., Pseudocrossidium crinitum (Schultz) R.H.Zander, Schistidium andinum (Mitt.) Herzog, S. praemorsum (Müll.Hal.) Herzog, Syntrichia lithophila (Dusén) Ochyra \& R.H.Zander y $S$. saxicola (Cardot) R.H.Zander. En sitios húmedos 
como bordes de arroyos y zonas pantanosas, son comunes Blindia magellanica Schimp., Breutelia integrifolia (Taylor) A.Jaeger, Bryum laevigatum Hook.f. \& Wilson, Dicranella hookeri (Müll. Hal.) Cardot, Syntrichia robusta (Hook. \& Grev.) R.H.Zander y Vittia pachyloma (Mont.) Ochyra.

\section{Conclusiones}

La diversidad de briófitos del Parque Nacional Torres del Paine antes de este estudio era desconocida, y aún no existe una lista patrón de los briófitos del parque.

En la ECP se puede encontrar una rica flora briofítica, totalizando 124 especies. Este número es aún preliminar pues el catastro se basa tan solo en 295 colecciones, sólo en las zonas aledañas a senderos habilitados. Las especies encontradas en la ECP representan solamente una fracción del total de la diversidad de especies de briófitas para el área geográfica que constituye el PNTP-ECP. Varios taxones reportados en la literatura para diferentes sectores del parque (e.g. Deguchi, 1984; Muñoz, 1999; Frey \& Schaumann, 2002; Townsend, 2006; Müller, 2009b; Larraín, 2012), y otros taxones depositados en los herbarios F, MO y SGO no fueron encontrados en el interior de la ECP. Este listado es un aporte para ser utilizado como punto de partida para estudios florísticos y vegetacionales tanto de la ECP como del PNTP, que incluyan la rica diversidad de briófitas.

Se han reportado entre 450 a 500 especies de plantas vasculares nativas en el PNTP (Vidal, 2007; Domínguez, 2012), además de 85 especies introducidas (Domínguez et al., 2006). Las briófitas forman parte importante dentro de las comunidades vegetales, las cuales han sido pobremente descritas. Aumentar el listado de especies vegetales para el área correspondiente a la ECP, es un precedente que indica que es necesario realizar prospecciones en el PNTP, con el fin de documentar toda la flora del parque.

La vulnerabilidad del PNTP al riesgo de incendios aumenta en la misma proporción en que aumenta el número de visitantes cada año. Esta condición ha dado paso a una serie de gestiones que han involucrado iniciativas de gobierno y privadas para aumentar las acciones de educación ambiental, aumentar los resguardos posibles de implementar en el parque, evaluar los daños, y proyectar los futuros trabajos de recuperación y reforestación (Vidal et al., 2015). Sin embargo sin información básica se hace imposible restaurar de manera exitosa, ya que se dejan de lado componentes fundamentales de los ecosistemas. Hablar de restauración implica dirigir los esfuerzos a favorecer distintos procesos bióticos y abióticos de los cuales se debe tener información previa para ser planificados y establecidos de manera adecuada. Actualmente se trabaja con modelos de referencia de áreas aledañas a los sectores incendiados y con información sobre la flora vascular del PNTP. Esta información se limita a la diversidad de flora vascular, sin embargo falta mucho conocimiento acerca de las distribuciones, abundancias, y de la biología de especies o de comunidades para poder contar con una base de información sólida para llevar a cabo procesos de restauración. Es menester incluir la totalidad de los organismos que forman parte de los ecosistemas si se pretende realizar líneas base que puedan ser útiles a la hora de conservar y/o restaurar los ecosistemas naturales.

\section{Agradecimientos}

Se agradece principalmente a Auna y Simón Larraín, por su gran ayuda en el trabajo de campo y por la recolección de interesante material, muchos de ellos nuevos registros para la provincia o para la región. A John Engel, Laura Briscoe y Blanka Shaw por su ayuda en la determinación de algunos grupos de hepáticas, María Jesús Cano y Juan Jiménez por su ayuda con algunas Pottiaceae, a Barbara Andreas por comentarios sobre la colección de Valdonia, y a Víctor Ardiles por entregar información acerca de las colecciones de briófitos de Torres del Paine conservados en el herbario SGO. Se agradece a Mauricio y Antonio Kusanovic de ECP por su interés en desarrollar este estudio, a AMA Torres del Paine por la planificación logística, y a Christian Andrade y Kavita Elliott por su ayuda en terreno.

\section{Bibliografía}

ARROYO, M. T. K., C. P. VON BOHLEN, L. CAVIERES \& C. MARTICORENA. 1992. Survey of the flora of Torres del Paine National Park, Chile. Gayana, Bot. 49: 47-70. 


\section{J. Larraín y N. Bahamonde - Briofitos de Torres del Paine, Chile}

BOELCKE, O., D. M. MOORE, \& F. A. ROIG (eds.). 1985. Transecta Botánica de la Patagonia Austral. Consejo Nacional de Investigación Científica y Técnica (Argentina), Buenos Aires.

BUCK, W. R. \& B. GOFFINET. 2010. Preliminary key to the mosses of Isla Navarino, Chile (Prov. Antártica Chilena). Nova Hedwigia, Beiheft 138: 213-227.

CALVELO, S., A. TREJO \& V. OJEDA. 2006. Botanical composition and structure of hummingbird nests in different habitats from northwestern Patagonia (Argentina). J. Nat. Hist. (London) 40: 589-603.

CARDOT, J. 1901. Mousses et Coup d'oeil sur la flore bryologique des Terres Magellaniques. En: Expedition Antarctique Belge. Résultats du Voyage du S.Y. Belgica en 1897-1898-1899 sous le commandement de A. de Gerlache de Gomery. Rapports Scientifiques. Botanique. Imprimerie J.-E. Buschmann, Anvers.

CARDOT, J. 1908. La flora bryologique des Terres magellaniques, de la Georgie du Sud et de l'Antarctide. Wissenschaftlichen Ergebnisse Schweidischen Sudpolar-Expedition 1901-1903, 4: 1-298.

CARDOT, J. \& V. F. BROTHERUS. 1923. Botanische ergebnisse der Schwedischen expedition nach Patagonien und dem Feuerlande 1907-1909. X. Les mousses. Kongliga Svenska Vetenskapsakademiens Handlingar 63: 1-74.

CORREA, M. N. (ed.). 1971-1999. Flora Patagónica. Partes I-VI. Colección Científica del INTA, Tomo VIII. Buenos Aires.

CONAF (Corporación Nacional Forestal). 2007. Plan de Manejo Parque Nacional Torres del Paine.

DEGUCHI, H. 1984. Studies on some Patagonian species of Grimmiaceae (Musci, Bryophyta). En: H. Inoue (ed.) Studies on Cryptogams in Southern Chile, pp. 17-72, Kensei-Sha, Tokyo.

DOLlENZ, O. 1991a. Capacidad de colonización de Rumex acetosella L. en comunidades perturbadas. An. Inst. Patagon. 20: 61-67.

DOLLENZ, O. 1991b. Sucesión vegetal en el sistema morrénico del glaciar Dickson, Magallanes, Chile. An. Inst. Patagon. 20: 49-60.

DOLLENZ, O. \& J. IVANOVIC. 1996. Sucesión secundaria en un pastizal incendiado en el Parque Nacional Torres del Paine, Magallanes, Chile. An. Inst. Patagon. 24: 15-28.

DOMÍNGUEZ, E. 2003. Orquídeas del Parque Nacional Torres del Paine. Revista Chile Forestal $N^{\circ} 300$. Documento Técnico 150: 1-11.

DOMÍNGUEZ, E. 2012. Flora Nativa. Torres del Paine. Ocho Libros Editores, Santiago.

DOMÍNGUEZ, E., C. MARTICORENA, A. ELVEBAKK \& A. PAUCHARD. 2004. Catálogo de la flora vascular del Parque Nacional Pali Aike. XII Región, Chile. Gayana, Bot. 61: 67-72.
DOMÍNGUEZ, E., A. ELVEBAKK, C. MARTICORENA $\&$ A. PAUCHARD. 2006. Plantas introducidas en el Parque Nacional Torres del Paine, Chile. Gayana, Bot. 63: 131-141.

DUSÉN, P. 1903. Beiträge zur Bryologie der Magellansländer, von Westpatagonien und Südchile. 1. Ark. Bot. 1: 441-466.

DUSÉN, P. 1905a. Beiträge zur Bryologie der Magellansländer, von Westpatagonien und Südchile. 2. Ark. Bot. 4 (1): 1-45.

DUSÉN, P. 1905b. Beiträge zur Bryologie der Magellansländer, von Westpatagonien und Südchile. 3. Ark. Bot. 4 (13): 1-24.

DUSÉN, P. 1906a. Beiträge zur Bryologie der Magellansländer, von Westpatagonien und Südchile. 4. Ark. Bot. 6 (8): 1-40.

DUSÉN, P. 1906b. Beiträge zur Bryologie der Magellansländer, von Westpatagonien und Südchile. 5. Ark. Bot. 6 (10): 1-32.

FREY, W. \& F. SCHAUMANN. 2002. Records of rare southern South American bryophytes. Studies in austral temperate rain forest bryophytes 18. Nova Hedwigia 74: 533-543.

GERSON, U. 1982. Bryophytes and Invertebrates. In: SMITH, A. J. E. (ed.), Bryophyte Ecology, pp. 291332. Chapman \& Hall, New York.

GOFFINET, B., R. ROZZI, L. LEWIS, W. R. BUCK \& F. MASSARDO. 2012. Miniature forests of Cape Horn/Los bosques en miniatura del Cabo de Hornos. Ecotourism with a hand lens/Ecotourismo con lupa. University of North Texas Press \& Ediciones Universidad de Magallanes, Chile.

GUNCKEL, H. 1971. Las primeras plantas herborizadas en Chile en 1690. An. Inst. Patagon. 2: 134-141.

HÄSSEL DE MENÉNDEZ, G., \& M. RUBIES. 2009. Catalogue of Marchantiophyta and Anthocerotophyta of southern South America Chile, Argentina and Uruguay, including Easter Is. (Pascua I.), Malvinas Is. (Falkland Is.), South Georgia Is., and the subantarctic South Shetland Is., South Sandwich Is., and South Orkney Is. Nova Hedwigia Beiheft 134: 1-672.

HOOKER, J. D., \& W. WILSON. 1844. Musci Antarctici. London J. Bot. 3: 533-556.

HONORATO, M. T., T. A. ALTAMIRANO, J. T. IBARRA, M. DE LA MAZA \& C. BONACIC. 2013. Composición de nidos de vertebrados nidificadores de cavidades en el bosque templado andino de Chile. V Reunión Binacional de Ecología, Puerto Varas, Chile.

HYVÖNEN, J. 1991. Tortella fragilis (Pottiaceae) reported for southern South America. Bryologist 96: 416-418.

LARRAÍN, J. 2012. Filogenia del género Racomitrium Brid. (Bryophyta, Grimmiaceae) y taxonomía de 
las especies latinoamericanas. Tesis doctoral, Universidad de Concepción, Chile. 293 pp.

LARRAÍN, J., \& C. MARTICORENA. 2009. Historia de la exploración briológica en Chile. Disponible en: www.musgosdechile.cl/historia.html [Acceso: 31 agosto 2015].

LEWINSKY, J. 1987. Orthotrichum (Orthotrichaceae) in South America 2. Taxonomic revision of taxa with superficial stomata. Mem. N. Y. Bot. Gard. 45: 326-370.

MATTERI, C. M. 1985. Catálogo de los musgos. In: BOELCKE, O., D. M. MOORE \& F. A. ROIG (eds.), Transecta botánica de la Patagonia austral, pp. 265-298., Consejo Nacional de Investigaciones Científicas y Técnicas (Argentina), Instituto de la Patagonia (Chile) and Royal Society, Buenos Aires

MÜLLER, C. 1885. Bryologia fuegiana. Flora 68: 391429.

MÜLLER, F. 2009a. An updated checklist of the mosses of Chile. Archive for Bryology 58: 1-124.

MÜLLER, F. 2009b. New records and new synonyms for the Chilean moss flora. Trop. Bryol. 30: 77-84.

MUÑOZ, J. 1999. A revision of Grimmia (Musci, Grimmiaceae) in the Americas. 1: Latin America. Ann. Mo. Bot. Gard. 86: 118-191.

OCHI, H. 1974. Some bryaceous 'Old World' mosses, also distributed in the New World. J. Fac. Educ. Tottori Univ., Nat. Sci. 25: 35-41.

OCHI, H. 1980. A revision of the neotropical Bryoideae, Musci (First part). J. Fac. Educ. Tottori Univ., Nat. Sci. 29: 49-154.

OCHYRA, R. 2003. Antipodal mosses: VIII. Valdonia gen. nov. (Seligeriaceae) from the Kerguelen Province in the Subantarctic. Cryptogam. Bryol. 24: 87-102.

OCHYRA, R., R. I. LEWIS SMITH \& H. BEDNAREKOCHYRA. 2008. The Illustrated Moss Flora of Antarctica. Cambridge University Press, New York.

PISANO, E. 1974. Estudios ecológicos de la región continental sur del área Andino-Patagónico. II: Contribución a la fitogeografía de la zona del "Parque Nacional Torres del Paine". An. Inst. Patagon. 5: 59-104.

PREUSSING, M., S. OLSSON, A. SCHÄFER-VERWIMP, N. J. WICKETT, S. WICKE, D. QUANDT \& M. NEBEL. 2010. New insights in the evolution of the liverwort family Aneuraceae (Metzgeriales, Marchantiophyta), with emphasis on the genus Lobatiriccardia. Taxon 59: 1424-1440.

RIELEY, J. O., P. W. RICHARDS \& A. D. L. BEBBINGTON. 1979. The ecological role of bryophytes in a North Wales woodland. J. Ecol. 67: 497-527.

ROIVAINEN, H. 1937. Bryological investigations in Tierra del Fuego. 1. Sphagnaceae-Dicranaceae. Arch. Soc. Zool. Bot. Fenn. Vanamo 9: I-X, 1-58.
ROSS, B. A., G. R. WEBSTER \& D. H. VITT. 1984. The role of mosses in reclamation of brine spills in forested areas. J. Can. Petrol. Technol. 23: 1-5.

ROZZI, R., L. LEWIS, F. MASSARDO, Y. MEDINA, K. MOSES, M. MÉNDEZ, L. SANCHO, P. VEZZANI, S. RUSSELL \& B. GOFFINET. 2012. Ecotourism with a hand lens in Omora Park. Ediciones Universidad de Magallanes, Punta Arenas.

SCHOFIELD, W. B. 1985. Introduction to Bryology. The Blackburn Press, New Jersey.

SCHUSTER, R. M. 1992. The Hepaticae and Anthocerotae of North America, east of the hundredth meridian. Vol. 5. Field Museum of Natural History, Chicago.

SUREN, A. 1993. Bryophytes and associated invertebrates in first-order alpine streams of Arthur's Pass, New Zealand. New Zeal. J. Mar. Fresh. 27: 479-494.

TORRES-DOWDALL, J., F. OSORIO \& G. SUÁREZ. 2007. Materiales utilizados por el picaflor rubí (Sephanoides sephaniodes) para la construcción de nidos en la selva Valdiviana, Chile. Ornitol. Neotrop. 18: 433-437.

TOWNSEND, C. C. 2006. Bryoerythrophyllum fuscinervium (Mitt.) R.H.Zander. In: BLOCKEEL et al., New national and regional bryophyte records, 12. J. Bryol. 28: 68-70.

VAN BREEMEN, N. 1995. How Sphagnum bogs down other plants. Trends Ecol. Evol. 10: 270-275.

VAN SETERS, T. E. \& J. S. PRICE. 2001. The impact of peat harvesting and natural regeneration on the water balance of an abandoned cutover bog, Quebec. Hydrol. Process. 15: 233-248.

VIDAL, O. J. 2007. Flora of Torres del Paine field guide. Editorial Fantástico Sur, Punta Arenas.

VIDAL, O.J. 2012. Torres del Paine, ecoturismo e incendios forestales: Perspectivas de investigación y manejo para una biodiversidad erosionada. Rev. Bosque Nat. 50: 33-39.

VIDAL, O. J., M. AGUAYO, R. NICULCAR, N. BAHAMONDE, S. RADIC, C. SAN MARTÍN, A. KUSCH, J. LATORRE \& J. FÉLEZ. 2015. Plantas invasoras en el Parque Nacional Torres del Paine (Magallanes, Chile): estado del arte, distribución post-fuego e implicancias en restauración ecológica. An. Inst. Patagon. 43: 75-96.

WILSON, W. \& J. D. HOOKER. 1847. Musci. In: Hooker, J. D. (eds.), The Botany of the Anarctic Voyage of H. M. Discovery Ships Erebus and Terror I. Flora Antarctica, Part II: Botany of Fuegia, The Falklands, Kerguelen's Land etc., pp. 395-551. Reeve Brothers, London.

Recibido el 11 de mayo de 2016, aceptado el 5 de septiembre de 2016. 